\title{
A multi-epitope-based vaccine designed by targeting cytoadherence proteins of Mycoplasma gallisepticum
}

Susithra Priyadarshni Mugunthan, ${ }^{\mathrm{a}}$

Mani Chandra Harish, ${ }^{\text {a }}$

a Plant Genetic Engineering and Molecular Farming Lab,

Department of Biotechnology, Thiruvalluvar University, Serkkadu, Vellore- 632115, Tamil

Nadu, India.

\section{*Corresponding Author:}

Assistant Professor, Department of Biotechnology, Thiruvalluvar University, Serkkadu, Vellore632115, Tamil Nadu, India.

Mobile: 09842726952

E-mail ID: mc.harish.tvu.edu.in 
Supplementary Table 1: List of predicted MHCI binding epitopes

\begin{tabular}{|c|c|c|c|c|c|c|}
\hline S.No & Protein & Allele & Peptide & $1-\log 50 \mathrm{k}(\mathrm{aff}) *$ & Affinity(nM)\# & \%Rank \\
\hline \multirow[t]{14}{*}{1.} & \multirow[t]{14}{*}{ GapA } & \multirow[t]{5}{*}{ HLA-B40:06 } & QEFTGFDAL & 0.417 & 549.34 & 0.50 \\
\hline & & & FEKEITAAV & 0.488 & 253.54 & 0.20 \\
\hline & & & KEITAAVNV & 0.493 & 242.26 & 0.17 \\
\hline & & & FENKINDIV & 0.439 & 431.64 & 0.40 \\
\hline & & & GEYVAVPQA & 0.581 & 93.27 & 0.05 \\
\hline & & \multirow[t]{5}{*}{ HLA-B41:04 } & QEFTGFDAL & 0.582 & 92.19 & 0.17 \\
\hline & & & LETQTTTPL & 0.530 & 162.13 & 0.40 \\
\hline & & & VESLFTTAL & 0.566 & 109.23 & 0.25 \\
\hline & & & KEITAAVNV & 0.516 & 187.13 & 0.50 \\
\hline & & & GEYVAVPQA & 0.518 & 184.30 & 0.40 \\
\hline & & \multirow[t]{4}{*}{ HLA-B41:03 } & QEFTGFDAL & 0.666 & 37.11 & 0.12 \\
\hline & & & $\begin{array}{l}\text { LETQTTTPL } \\
\end{array}$ & 0.672 & 34.77 & 0.10 \\
\hline & & & VESLFTTAL & 0.682 & 31.21 & 0.07 \\
\hline & & & FEKEITAAV & 0.621 & 60.71 & 0.30 \\
\hline \multirow[t]{7}{*}{2.} & \multirow[t]{7}{*}{$\mathrm{PlpA}$} & \multirow[t]{2}{*}{ HLA-B40:06 } & YEYPEYEEI & 0.427 & 492.48 & 0.40 \\
\hline & & & LENQYYPPA & 0.516 & 188.31 & 0.50 \\
\hline & & \multirow[t]{3}{*}{ HLA-B41:03 } & YEYPEYEEI & 0.601 & 74.57 & 0.50 \\
\hline & & & IESQLQALL & 0.649 & 44.84 & 0.17 \\
\hline & & & FEKLMLANM & 0.610 & 68.01 & 0.40 \\
\hline & & \multirow[t]{2}{*}{ HLA-B41:04 } & IESQLQALL & 0.503 & 216.28 & 0.50 \\
\hline & & & FEKLMLANM & 0.513 & 193.54 & 0.50 \\
\hline
\end{tabular}




\begin{tabular}{|c|c|c|c|c|c|c|}
\hline & & & LENQYYPPA & 0.516 & 187.77 & 0.50 \\
\hline \multirow[t]{12}{*}{3.} & \multirow[t]{12}{*}{ Hlp3 } & \multirow[t]{4}{*}{ HLA-B40:06 } & AEVQPEPVV & 0.435 & 451.94 & 0.40 \\
\hline & & & QEYDYYPPA & 0.583 & 91.54 & 0.50 \\
\hline & & & RQLGMIEPV & 0.413 & 572.43 & 0.50 \\
\hline & & & AEFRSRTLA & 0.490 & 250.27 & 0.20 \\
\hline & & \multirow[t]{3}{*}{ HLA-B41:03 } & QELLRYNVI & 0.602 & 74.16 & 0.50 \\
\hline & & & AEQVEQQPV & 0.625 & 57.82 & 0.30 \\
\hline & & & QETKHHHAL & 0.663 & 38.54 & 0.12 \\
\hline & & \multirow[t]{5}{*}{ HLA-B41:04 } & QELLRYNVI & 0.588 & 86.16 & 0.17 \\
\hline & & & AEQVEQQPV & 0.545 & 137.63 & 0.30 \\
\hline & & & QETKHHHAL & 0.560 & 117.14 & 0.25 \\
\hline & & & QEYDYYPPA & 0.538 & 147.88 & 0.40 \\
\hline & & & AEFRSRTLA & 0.514 & 192.48 & 0.50 \\
\hline \multirow[t]{5}{*}{4.} & \multirow[t]{5}{*}{ CrmA } & HLA-B40:06 & NEIGVILPL & 0.518 & 184.51 & 0.12 \\
\hline & & \multirow[t]{2}{*}{ HLA-B41:03 } & NEIGVILPL & 0.693 & 27.86 & 0.05 \\
\hline & & & GEIVNDFIL & 0.621 & 60.60 & 0.30 \\
\hline & & \multirow[t]{2}{*}{ HLA-B41:04 } & NEIGVILPL & 0.594 & 80.45 & 0.15 \\
\hline & & & RQTRAYYAL & 0.591 & 83.36 & 0.17 \\
\hline
\end{tabular}

Supplementary Table 2: List of MHCII binding epitopes

\begin{tabular}{|l|l|l|l|l|l|l|l|}
\hline $\begin{array}{l}\text { S.N } \\
\text { o }\end{array}$ & $\begin{array}{l}\text { Prote } \\
\text { in }\end{array}$ & Allele & Peptide & Core peptide & $\begin{array}{l}\mathbf{1 -} \\
\operatorname{log50k(aff} \\
)^{*}\end{array}$ & $\begin{array}{l}\text { Binding } \\
\text { Affinity(n } \\
\text { M)\# }\end{array}$ & $\begin{array}{l}\text { \%Ra } \\
\text { nk }\end{array}$ \\
\hline 1. & GapA & DRB1_1 & IRLRLLVIDRSRAT & LLVIDRSRA & 0.660 & 39.48 & 0.09 \\
\hline
\end{tabular}




\begin{tabular}{|c|c|c|c|c|c|c|c|}
\hline & & 482 & $\mathrm{~N}$ & & & & \\
\hline & & $\begin{array}{l}\text { DRB1_1 } \\
445\end{array}$ & $\begin{array}{l}\text { IRLRLLVIDRSRAT } \\
\mathrm{N}\end{array}$ & LLVIDRSRA & 0.560 & 117.07 & 0.12 \\
\hline & & & $\begin{array}{l}\text { NPRNILNALTTAK } \\
\text { GF }\end{array}$ & LNALTTAKG & 0.464 & 328.57 & 2.00 \\
\hline & & & $\begin{array}{l}\text { PFEKEITAAVNVR } \\
\text { SL }\end{array}$ & ITAAVNVRS & 0.462 & 336.19 & 2.00 \\
\hline & & $\begin{array}{l}\text { DRB1_1 } \\
366\end{array}$ & $\begin{array}{l}\text { IRLRLLVIDRSRAT } \\
\mathrm{N}\end{array}$ & LLVIDRSRA & 0.780 & 10.84 & 0.40 \\
\hline & & $\begin{array}{l}\text { DRB1_1 } \\
310\end{array}$ & $\begin{array}{l}\text { IRLRLLVIDRSRAT } \\
\mathrm{N}\end{array}$ & LLVIDRSRA & 0.731 & 18.29 & 0.70 \\
\hline 2. & PlpA & $\begin{array}{l}\text { DRB1_1 } \\
445\end{array}$ & $\begin{array}{l}\text { KTFSLNKGLNKVII } \\
\text { R }\end{array}$ & LNKGLNKVI & 0.467 & 320.42 & 1.80 \\
\hline & & $\begin{array}{l}\text { DRB1_1 } \\
310\end{array}$ & $\begin{array}{l}\text { KTFSLNKGLNKVII } \\
\text { R }\end{array}$ & LNKGLNKVI & 0.700 & 25.58 & 1.60 \\
\hline 3. & Hlp3 & $\begin{array}{l}\text { DRB1_1 } \\
482\end{array}$ & $\begin{array}{l}\text { KIHNKILKNLAKL } \\
\text { KK }\end{array}$ & ILKNLAKLK & 0.601 & 75.28 & 0.60 \\
\hline & & & $\begin{array}{l}\text { NQTFNLIKPVFKFL } \\
\text { K }\end{array}$ & FNLIKPVFK & 0.556 & 121.44 & 1.80 \\
\hline & & & $\begin{array}{l}\text { RPTYGLRRVSRIPS } \\
\text { L }\end{array}$ & LRRVSRIPS & 0.556 & 121.99 & 1.80 \\
\hline & & $\begin{array}{l}\text { DRB1_1 } \\
445\end{array}$ & $\begin{array}{l}\text { KIHNKILKNLAKL } \\
\text { KK }\end{array}$ & ILKNLAKLK & 0.523 & 173.55 & 0.40 \\
\hline & & & $\begin{array}{l}\text { RPTYGLRRVSRIPS } \\
\text { L }\end{array}$ & LRRVSRIPS & 0.470 & 309.43 & 1.70 \\
\hline & & $\begin{array}{l}\text { DRB1_1 } \\
366\end{array}$ & $\begin{array}{l}\text { KIHNKILKNLAKL } \\
\text { KK }\end{array}$ & ILKNLAKLK & 0.782 & 10.61 & 0.40 \\
\hline & & & $\begin{array}{l}\text { QTFNLIKPVFKFLK } \\
\mathrm{K}\end{array}$ & FNLIKPVFK & 0.731 & 18.28 & 1.90 \\
\hline
\end{tabular}




\begin{tabular}{|c|c|c|c|c|c|c|c|}
\hline & & & $\begin{array}{l}\text { YGLRRVSRIPSLAP } \\
\text { R }\end{array}$ & LRRVSRIPS & 0.732 & 18.15 & 1.90 \\
\hline & & $\begin{array}{l}\text { DRB1_1 } \\
310\end{array}$ & $\begin{array}{l}\text { KIHNKILKNLAKL } \\
\text { KK }\end{array}$ & ILKNLAKLK & 0.728 & 19.06 & 0.80 \\
\hline \multirow[t]{9}{*}{4.} & \multirow[t]{9}{*}{ CrmA } & \multirow[t]{4}{*}{$\begin{array}{l}\text { DRB1_1 } \\
445\end{array}$} & $\begin{array}{l}\text { FSRLYLNSVNSLSF } \\
\text { I }\end{array}$ & LYLNSVNSL & 0.477 & 286.75 & 1.40 \\
\hline & & & $\begin{array}{l}\text { ATTYLRSQIGLAR } \\
\text { TS }\end{array}$ & LRSQIGLAR & 0.468 & 317.79 & 1.80 \\
\hline & & & PGDQFSSIKNIRTIF & FSSIKNIRT & 0.471 & 305.49 & 1.70 \\
\hline & & & $\begin{array}{l}\text { SVYKKIITQTANV } \\
\text { KK }\end{array}$ & IITQTANVK & 0.464 & 328.38 & 2.00 \\
\hline & & \multirow[t]{2}{*}{$\begin{array}{l}\text { DRB1_1 } \\
366\end{array}$} & $\begin{array}{l}\text { FSRLYLNSVNSLSF } \\
\text { I }\end{array}$ & LYLNSVNSL & 0.743 & 16.09 & 1.40 \\
\hline & & & PGDQFSSIKNIRTIF & FSSIKNIRT & 0.744 & 16.00 & 1.40 \\
\hline & & \multirow[t]{3}{*}{$\begin{array}{l}\text { DRB1_1 } \\
310\end{array}$} & $\begin{array}{l}\text { FSRLYLNSVNSLSF } \\
\text { I }\end{array}$ & LYLNSVNSL & 0.730 & 18.57 & 0.70 \\
\hline & & & $\begin{array}{l}\text { EIVNDFILDAPILP } \\
\mathrm{K}\end{array}$ & FILDAPILP & 0.631 & 54.22 & 1.80 \\
\hline & & & $\begin{array}{l}\text { LGFDGIRNNLNVG } \\
\text { VK }\end{array}$ & IRNNLNVGV & 0.654 & 42.06 & 1.10 \\
\hline
\end{tabular}


Supplementary Table 3: Models from Galaxy refine server

\begin{tabular}{|l|c|c|c|c|c|c|}
\hline Model & GDT-HA & RMSD & MolProbity & Clash score & $\begin{array}{l}\text { Poor } \\
\text { rotomers }\end{array}$ & $\begin{array}{l}\text { Rama } \\
\text { favored }\end{array}$ \\
\hline MODEL 1 & 0.7832 & 0.820 & 4.049 & 126.8 & 5.7 & 60.8 \\
\hline MODEL 2 & 0.7883 & 0.821 & 4.052 & 117.5 & 6.3 & 60.8 \\
\hline MODEL 3 & 0.7793 & 0.838 & 4.100 & 122.3 & 7.0 & 60.8 \\
\hline MODEL 4 & 0.7908 & 0.828 & 4.127 & 117.2 & 8.2 & 62.4 \\
\hline MODEL 5 & 0.7806 & 0.822 & 3.998 & 124.3 & 5.1 & 61.3 \\
\hline
\end{tabular}

Supplementary Table 4: The binding energy and attractive VDW scores for suggested epitopes with chicken BF2 alleles using PatchDock

\begin{tabular}{|c|c|c|c|c|c|}
\hline S.No & Protein & Ligand & Receptor & $\begin{array}{l}\text { Global Energy } \\
\text { kcal/mol }\end{array}$ & $\begin{array}{l}\text { Attractive VDW } \\
\text { kcal/mol }\end{array}$ \\
\hline \multirow[t]{2}{*}{1.} & GapA & QEFTGFDAL & $\begin{array}{l}\text { BF2 } \\
2101\end{array}$ & -44.69 & -20.36 \\
\hline & & & $\begin{array}{l}\text { BF2 } \\
0401\end{array}$ & -6.25 & -4.75 \\
\hline \multirow[t]{2}{*}{2.} & PlpA & YEYPEYEEI & $\begin{array}{l}\text { BF2 } \\
2101\end{array}$ & -58.07 & -27.10 \\
\hline & & & $\begin{array}{l}\text { BF2 } \\
0401\end{array}$ & -46.58 & -22.33 \\
\hline \multirow[t]{2}{*}{3.} & Hlp3 & QELLRYNVI & $\begin{array}{l}\text { BF2 } \\
2101\end{array}$ & -44.73 & -26.81 \\
\hline & & & $\begin{array}{l}\text { BF2 } \\
0401\end{array}$ & -44.05 & -29.36 \\
\hline
\end{tabular}




\begin{tabular}{|c|c|c|c|c|c|}
\hline \multirow[t]{2}{*}{4.} & CrmA & \multirow[t]{2}{*}{ NEIGVILPL } & $\begin{array}{l}\text { BF2 } \\
2101\end{array}$ & -69.39 & -27.93 \\
\hline & & & $\begin{array}{l}\text { BF2 } \\
0401\end{array}$ & -48.16 & -29.74 \\
\hline
\end{tabular}
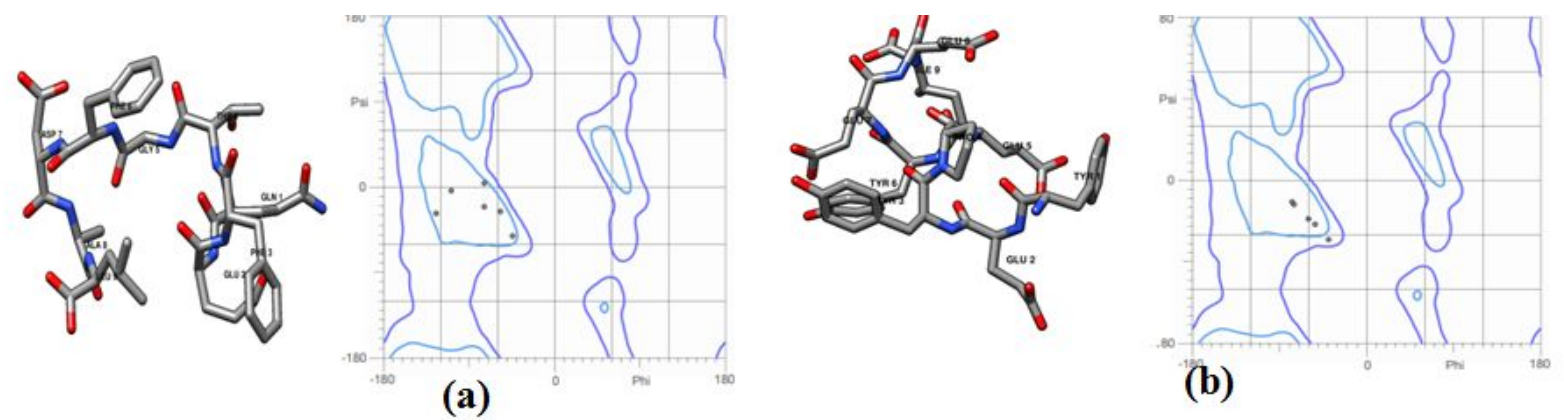

(b)

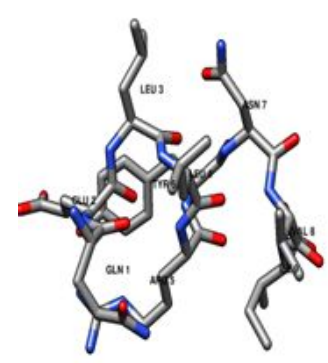

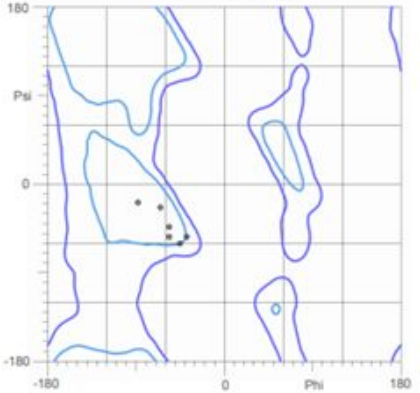

(c)

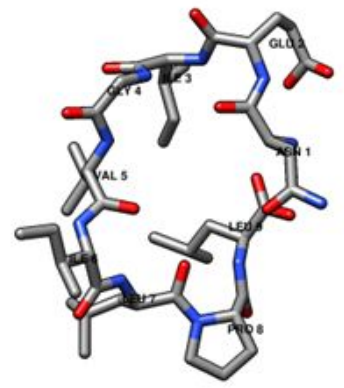

(d)

Supplementary Figure 1: Model and structure validation of epitopes (a) GapA (QEFTGFDAL), (b) PlpA (YEYPEYEEI), (c) Hlp3 (QELLRYNVI), (d) CrmA (NEIGVILPL). 

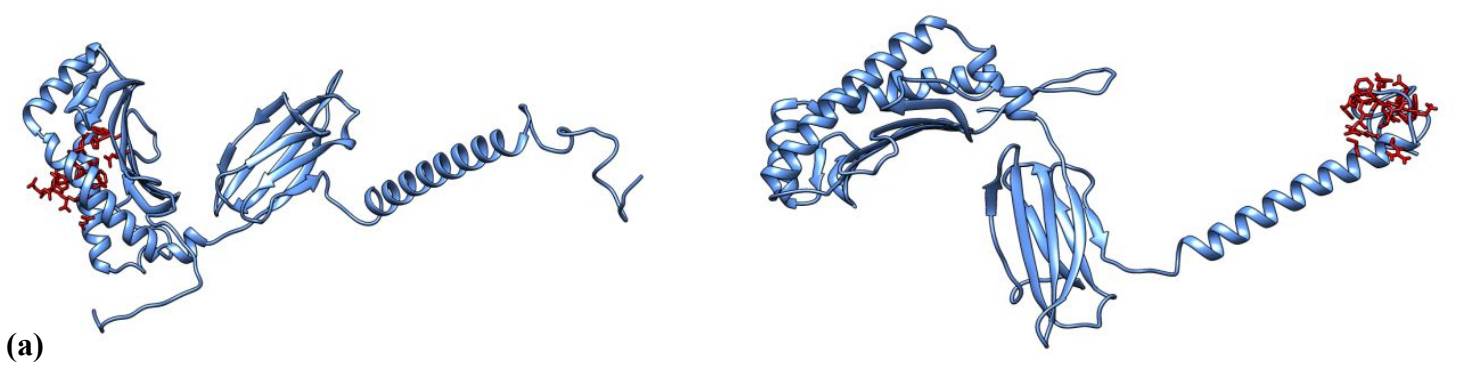

(b)
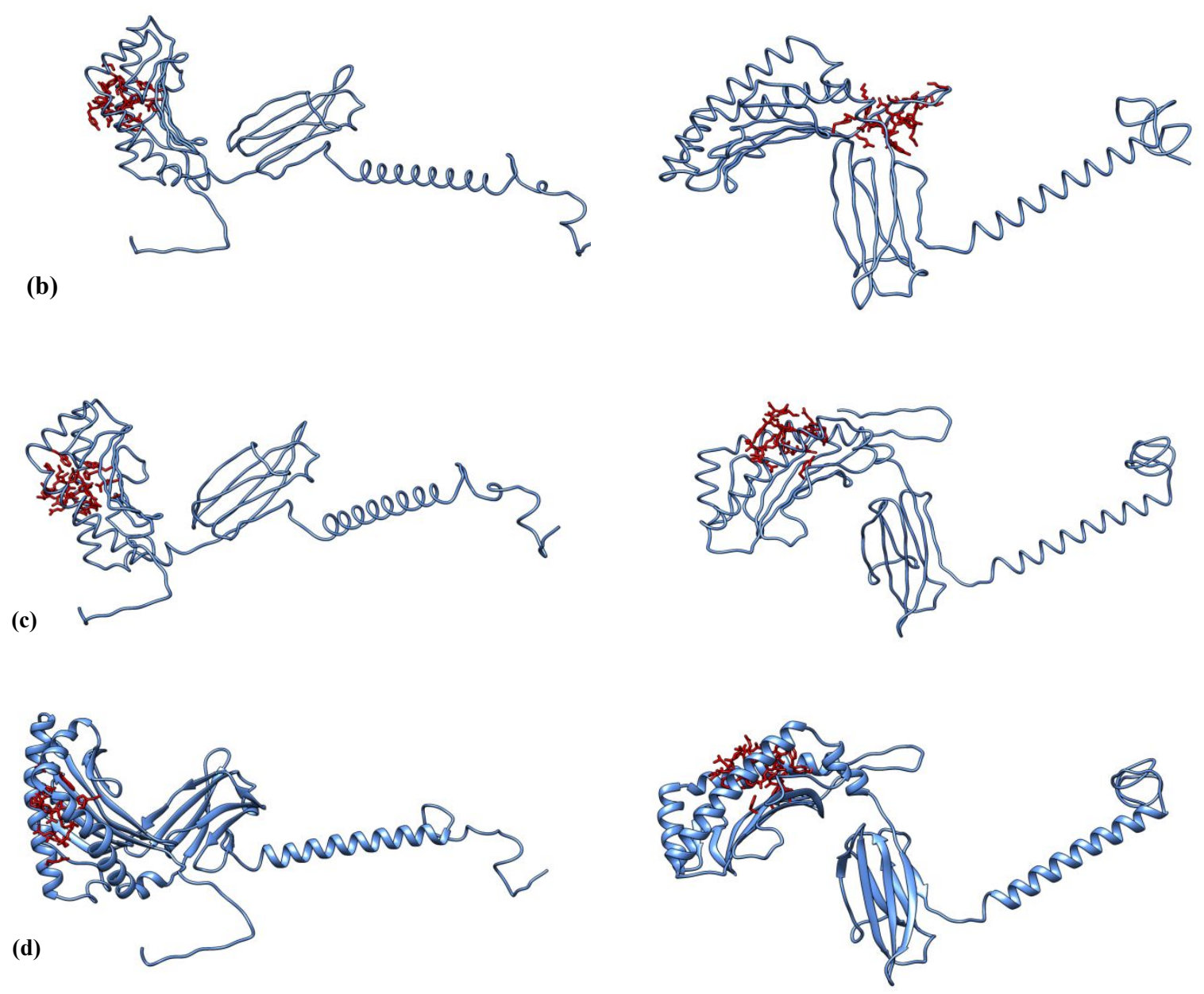

Supplementary Figure 2: Docked complex of predicted epitopes with BF2 2101 and BF2 0401 alleles respectively. (a) GapA, (b) PlpA, (c) Hlp3, (d) CrmA 